Communications in Physics, Vol. 24, No. 3S1 (2014), pp. 7-12

DOI:10.15625/0868-3166/24/3S1/5071

\title{
ENHANCED PERFORMANCE IN POLYMER LIGHT EMMITING DIODE BY USING ULTRA-THIN CONDUCTIVE FILMS AS THE BUFFER LAYER
}

\author{
NGUYEN PHUONG HOAI NAM \\ Faculty of Engineering Physics and Nano-Technology, University of Engineering and \\ Technology, Vietnam National University, Hanoi \\ E-mail: namnph@vnu.edu.vn
}

Received 20 June 2014

Accepted for publication 20 August 2014

\begin{abstract}
The ultra-thin nanocomposite films based on the nano-crystal $\mathrm{TiO}_{2}\left(\mathrm{TiO}_{2}-\mathrm{nc}\right)$ or multi-walled carbon nanotube (MWCNTs) were prepared and used as the buffer layers in the fabrication of the organic light-emitting diodes (OLEDs). The injection efficiency of the hole and electron was improved by inserting an ultra-thin buffer layer between the electrodes and emissive layer. The turn-on voltage of the device with the buffer layers was lowered from 4 to $2.5 \mathrm{~V}$, and the current density was increased from 0.3 to $0.7 \mathrm{~mA} / \mathrm{mm}^{2}$, in comparison with the device without such a buffer layer. These devices showed a high efficiency and good stability.
\end{abstract}

Keywords: nanocomposites, conducting polymer, blended polymer, light emitting diode, CNTs. .

\section{INTRODUCTION}

Organic light-emitting diodes (OLEDs) were frequently applied to flat panel displays due to the ease of manufacturing, solid-state, faster switching speed and wider viewing angle, etc... Along with the developing of new technology, OLEDs have the potential to substitute the liquid crystal display (LCDs) and become the pacemaker in the display market. High performance organic light emitting diodes should have a low operating voltage, high efficiency and relatively good stability. In order to improve the efficiency of devices, various techniques are available as anode or cathode modification, annealing and optical coupling [1 - 4]. For example, cathode modification has been shown to increase the electron injection, so as to improve the electron-hole balance. As the result, the efficiency of the devices can be improved. The electroluminescence efficiencies of organic light emitting diodes can also be promoted with a better charge injection as well as the balance of the opposite charge carriers. It is widely recognized that unbalanced charge carriers due to a higher hole mobility in the hole transporting layer (HTL) and slower electron mobility in the electron transporting layer (ETL) leads to reduced efficiency of OLEDs. It is thus important to balance the injected charges to improve device performance. Recently, much work has been made on device structure especially on the interface of device $[5,6]$. Some organic materials and inorganic insulating materials have been adopted as hole injection buffer layers inserted between the indium tin oxide (ITO) anode and the organic layer, such as copper phthalocyanine (CuPc), 
polyaniline, $\mathrm{SiO}_{2}, \mathrm{Al}_{2} \mathrm{O}_{3}$, and so on [7 - 11]. In this work, the ultra-thin conductive film based on poly (3,4-ethylenedioxythiophene) poly (styrenesulfonate) (PEDOT-PSS) and multi walled carbon nano-tubes (MWCNTs) as well as $\mathrm{TiO}_{2}$ nano-crystal $\left(\mathrm{TiO}_{2}-\mathrm{nc}\right)$ have been fabricated and used as the buffer layers. Some kinds of organic light emitting diode (OLED) have been fabricated and the devices properties were compared with each other. The ultra-thin films of PEDOT-PSS:MWCNTs and $\mathrm{TiO}_{2}$-nc were inserted at the interface of one anode (ITO)/the emitting layer (PVK:MEH-PPV) and the cathode $(\mathrm{Al}) /$ the emitting layer, respectively. It provides an improved efficiency and good stability as compared to the control device. The blend films of poly [9-vinylcarbarzole] (PVK) and poly [2-methoxy-5-(2-ethylhexyloxy)-1, 4-phenylenevinylene] (MEH-PPV) with optimal weight ratios of PVK:MEH-PPV have been fabricated and used as the emitting layer. The energy transfer process from PVK to MEH-PPV was observed, and thus the emission of MEH-PPV was exclusively observed when the blended polymer film was photo-excited by light whose energy was corresponding to the absorption of PVK. The current density - voltage $(\mathrm{J}-\mathrm{V})$ characteristics of the devices were also studied.

\section{EXPERIMENTAL}

In this study, the model devices have been fabricated and their properties were studied and compared. The $\mathrm{TiO}_{2}$-nc thin film was introduced between the emission layer and the cathode. The PEDOT-PSS:MWCNTs thin film was used as anode buffer layer. The device configuration of ITO/PEDOT-PSS:CNTs/PVK:MEH$\mathrm{PPV} / \mathrm{TiO}_{2}$-nc/Al is showed in Fig. 1.

The MWCNTs, supplied from Shenzhen Nanotech Port Co. Ltd., were further purified with strong acid to remove the impurities. The acid-modification of CNTs was based on a mixture of concentrated nitric and sulfuric acids in

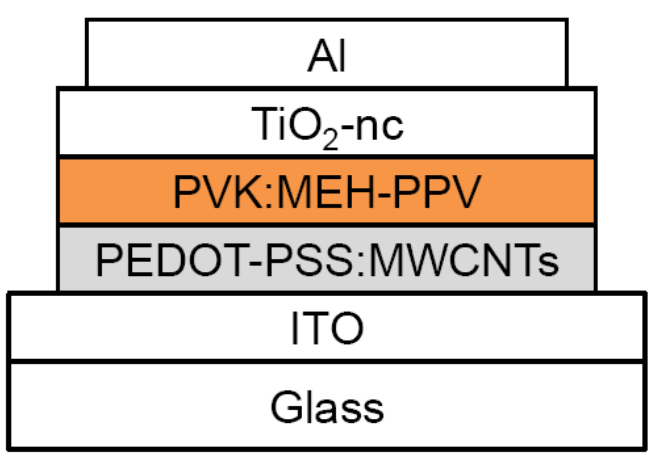

Fig. 1. The structure of the OLED. a molar ratio of $3: 1$, respectively [12]. The $\mathrm{TiO}_{2}$ nanoparticles were available as an aqueous solution of a $10 \mathrm{wt}$. \% suspension of $\mathrm{TiO}_{2}$ in $\mathrm{H}_{2} \mathrm{O}$ (purchased from Sigma-Aldrich). The $\mathrm{TiO}_{2}$ nano-cluster films were prepared by spin-coating at 3000 rpm to investigate the effect of the electrodes buffer layers on the performance of the devices. The conducting polymers PVK and MEH-PPV were purchased from Aldrich Chemical Co. and used as received. Indium tin oxide (ITO) and Al were used as the anode and the cathode, respectively. The sheet resistance of the ITO was $25 \Omega \mathrm{cm}^{-1}$. Before using, the ITO substrate and glass were routinely cleaned by ultra-sonication in mixture of acetone and alcohol, alcohol and de-ionized water [1]. The blended polymers were obtained by mixing PVK with MEH-PPV (PVK:MEH$\mathrm{PPV}=100: 15)$ [13] and then the blends were spin-coated onto the substrates and dried in vacuum at $80^{\circ} \mathrm{C}$ for two hours. The thickness of the polymer layers were controlled both by spin speed and by the concentration of the polymers in solvent. The film thickness was measured by using a $\alpha$ step DEKTAK and to be around $120 \mathrm{~nm}$. The surface morphology of the PEDOT-PSS:MWCNTs nano-composite thin films were investigated by using a Hitachi Field Emission Scanning Electron 
Microscopy (FE-SEM) S-4800 and the surface resistivity of these films were measured by fourprobe resistivity measurements (JANDEL - UK) at room temperature. The transmittance spectra of the nano-composite thin films were attained from a Jasco UV-VIS-NIR V570 spectrometer. The current density - voltage $(\mathrm{J}-\mathrm{V})$ characteristics of the devices were measured on an Auto-Lab Potentiostat PGS-30. All the photo-physical measurements were performed at room temperature in air.

\section{RESULTS AND DISCUSSION}

The nano-composites were obtained by mixing the acid-treated MWCNTs and PEDOT-PSS (PEDOT-PSS:MWCNTs) in the ratios of 100:0.5 (weight fraction). The thin films was prepared by spin-coating onto glass subtrate and dried in vacuum at $80^{\circ} \mathrm{C}$ for two hours. Fig. 2 shows the FE-SEM images of the PEDOT-PSS:MWCNTs nano-composite thin film. It can be seen that the MWCNTs are distributed fairly uniformly. The Fig.2 also shows that the MWCNTs have a diameter of $30 \mathrm{~nm}$ and their lengths of $300-500 \mathrm{~nm}$ (Fig.2a) and the thickness of the film of about $25 \mathrm{~nm}$ (Fig.2b).

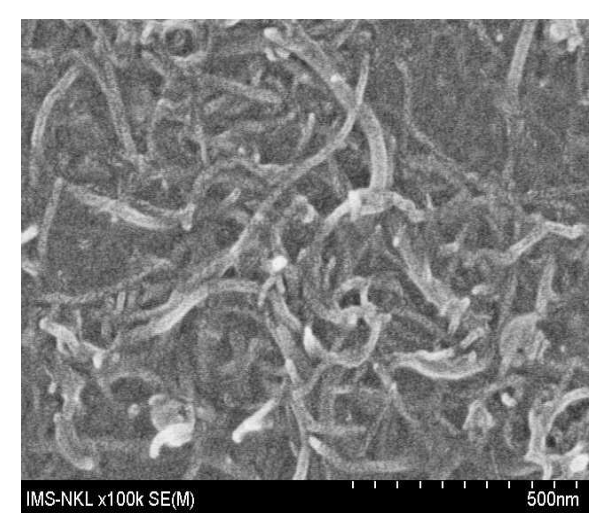

(a)

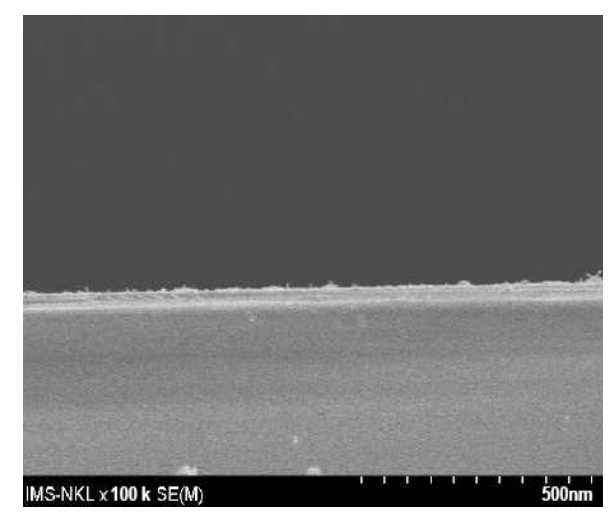

(b)

Fig. 2. FESEM of surface (a) and cross (b) of PEDOT-PSS:MWCNTs thin films.

The transmittance spectra of the nano-composite thin films in a range from near ultraviolet to near infrared light are shown in Fig.3. The nano-composite film of PEDOT-PSS:MWCNTs = 100:0,5 (weight fraction) shows the transmittance of about $80 \%$.

The resistance of the films decreases rapidly when increasing MWCNTs concentration and goes to minimum value of $36.5 \Omega . \mathrm{sq}^{-1}$ at PEDOT-PSS:MWCNTs $=100: 0.5$ (weight fraction) due to the excellent conduction of the MWCNTs. However, the resistance of the films increases back when MWCNTs concentration exceeds the optimal value (PEDOT-PSS:MWCNTs = 100:0.5). The increase of the resistance of the films can be attributed to the increase of the defects of the films when the concentration of MWCNTs reached higher than the optimal point.

Fig. 4 is the FE-SEM image of the surface of the $\mathrm{TiO}_{2}$-nc film. It can be seen that the distribution of the $\mathrm{TiO}_{2}$ nanoparticles with $30-40 \mathrm{~nm}$ in diameter was fairly uniform. 


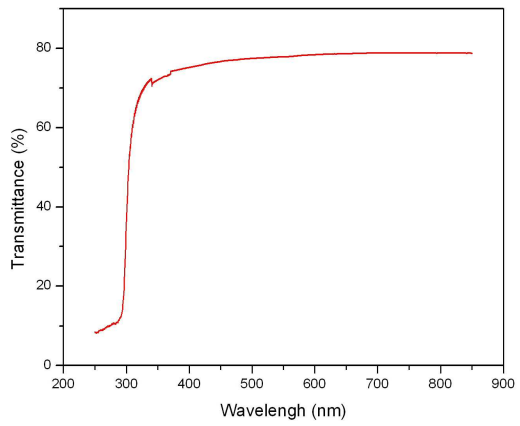

Fig. 3. Transmittance of the nano-composite thin films of PEDOT-PSS:MWCNTs.

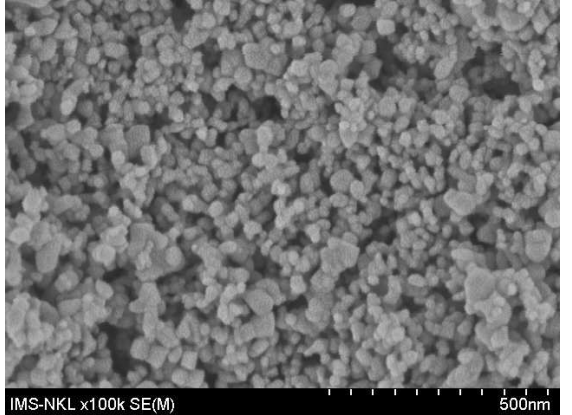

Fig. 4. FE-SEM of the $\mathrm{TiO}_{2}$-nc film.

Fig. 5 compares the photoluminescence (PL) spectra of the bulk films of PVK and PVK+MEHPPV. The PL emission from PVK film excited at $325 \mathrm{~nm}$ overlaps with the absorption peaks of MEH-PPV, and thus an efficient Förster energy transfer can be anticipated [13].

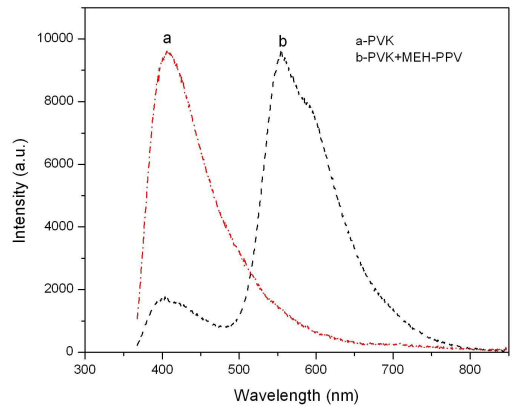

(a)

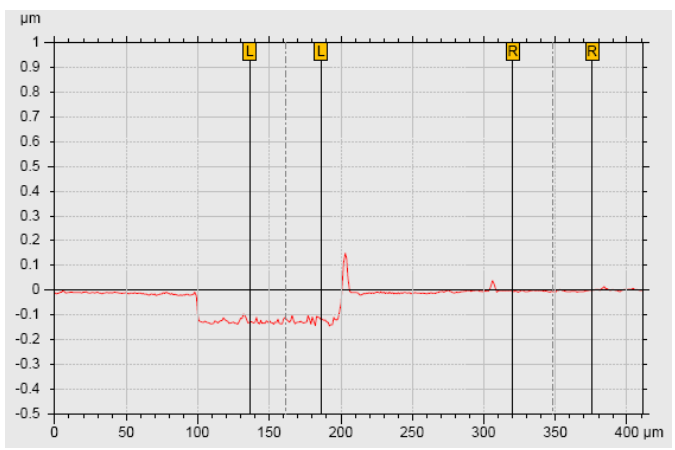

(b)

Fig. 5. Photoluminescence spectra excited at $325 \mathrm{~nm}$ (a) and the thickness of the films measureing by $\alpha$ step DEKTAK and to be around $120 \mathrm{~nm}$.

Fig. 6 shows the current density - voltage $(\mathrm{J}-\mathrm{V})$ characteristics of the single layer device (A) and the multilayer device using the PEDOT-PSS and PEDOT-PSS:MWCNTs films as the anode buffer layer (B and C). The multilayer device were fabricated consisting of a transparent indium-tin oxide (ITO) electrode, the ultra thin film as buffer layer which has a thickness of 25 $\mathrm{nm}$, the blend conducting polymer film and an aluminum (Al) electrode.

From Fig. 6 we can see that the $J-V$ performances of the devices that are strongly dependent on the presence of the nano-composite thin film PEDOT-PSS:MWCNTs between the anode and the emitting layer. It can be seen that the current density of the multilayer devices (B and C) are much higher compared with those of the single layer device (A) at the same operating voltage. Also, the threshold field of the multilayer devices is decreased strongly to lower values than $3 \mathrm{~V}$. The single layer device performed very poorly. This result suggests that the tunneling of charge 


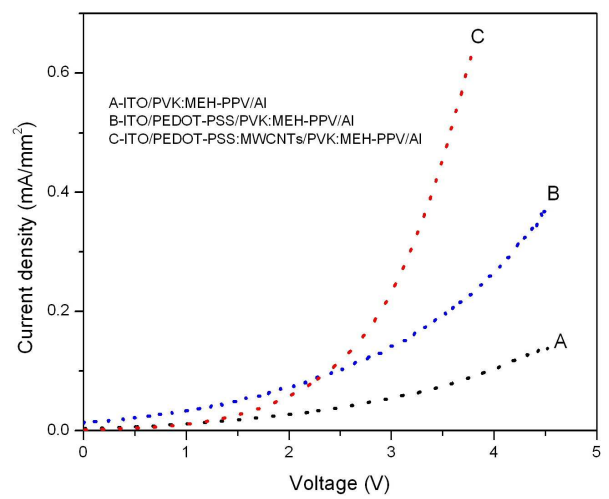

Fig. 6. The J-V characteristics of the devices using PEDOT-PSS:MWCNTs film.

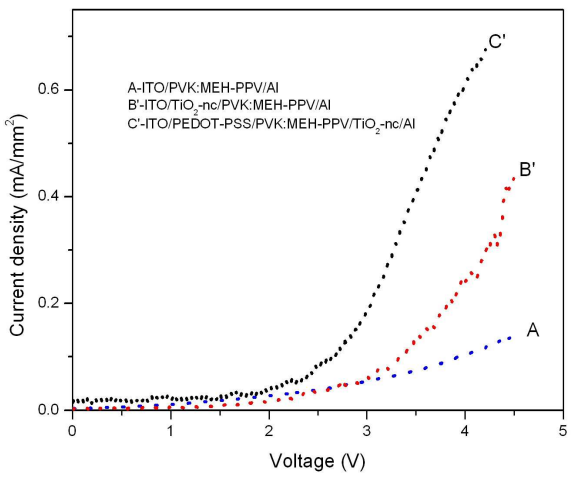

Fig. 7. The J-V characteristics of the devices using $\mathrm{TiO}_{2}$-nc film.

carriers between ITO and PVK:MEH-PPV can highly enhance the injection of holes due to the large potential drop across a ultra-thin conducting layer. Hence, the turn-on voltage is reduced and overall current density is increased [14]. But it shows that the bias voltage to obtain the same current density is obviously increased for the OLEDs with PEDOT-PSS buffer layer compared with the device with PEDOT-PSS:MWCNTs buffer layer. This is probably because the hole injection ability of the PEDOT-PSS might be improved by the MWCNTs fillings [15]. As the result, the PEDOT-PSS:MWCNTs thin layer enhances most of the holes injected from the anode to the emitting layer (PVK:MEH-PPV). In addition, The decrease of the resistance of the nano-composites PEDOT-PSS:MWCNTs will reduce the bulk resistance of the OLEDs, which might attribute to the increase of the current density. Therefore, a great improvement in the performances of the devices was achieved with the nano-composites of PEDOT-PSS and MWCNT as hole injection layer of the OLEDs.

Fig. 7 shows the current density - voltage (J-V) characteristics of a single device $(A)$ and the multi-device using the $\mathrm{TiO}_{2}$-nc films as the anode buffer layer (B') and the cathode buffer layer (C'). The thickness of the ultra thin $\mathrm{TiO}_{2}$-nc film was estimated to be around $20-30 \mathrm{~nm}$.

From Fig. 7 we see that the J-V performances of the devices are strongly dependent on the presence of the $\mathrm{TiO}_{2}$-nc film between the anode and the emitting layer (device B') and the cathode and the emitting layer (device $C^{\prime}$ ). It can be seen that the current density of the multi layer devices (B' and C') are much higher compared with those of the single device (A) at the same operating voltage. The turn-on voltage of the multi devices is decreased to lower values than $3 \mathrm{~V}$. The device which combined $\mathrm{TiO}_{2}$-nc thin film as cathode buffer layer and PEDPT-PSS as anode buffer layer, respectively, shows the best performance with a turn-on voltage about $2.5 \mathrm{~V}$ and the current density at $0.7 \mathrm{~mA} / \mathrm{mm}^{2}$ (device C'). The improvements of the performance of the device can be considered in order to explain the behavior of the nanoparticle $\mathrm{TiO}_{2}-$ modified devices. The hole mobility in ordinary PPV is two orders of magnitude higher than the electron mobility [16], resulting in a recombination zone that is very close to the aluminum cathode. In addition the barrier for hole injection is lower than the barrier for electron injection. Hence, the $\mathrm{J}-\mathrm{V}$ characteristics of the device is mainly determined by the holes [17]. The nanoparticle $\mathrm{TiO}_{2}$, 
arbitrarily distributed between the PVK:MEH-PPV and the aluminum layer, create a randomly nano-patterned cathode interface. This gives rise to locally enhanced fields again resulting in a higher electron injection rate, in turn leading to a better charge balance. The enhanced internal quantum efficiency entails finally an increased luminescence. This interpretation is supported by the lower turn-on voltage and the high enhancement factor at low current densities.

\section{CONCLUSION}

We have fabricated OLEDs with the ultra thin films between the emission layer and the electrodes as the buffer layers. The performance of the device is improved in decreasing turn-on voltage (to $2.5 \mathrm{~V}$ ) and increasing current density (to $0.7 \mathrm{~mA} / \mathrm{mm}^{2}$ ), leading to an increase in the luminance and efficiency of the device. The nano-composites of PEDOT:PSS and MWCNTs were fabricated by inclusion of acid-treated MWCNTs into aqueous PEDOT:PSS solution. The nanocomposite increase the hole injection at the nano-composite - anode interface therefore enhancing the internal quantum efficiency. The metal or semimetal properties of the MWCNTs might contribute to the improvement of the nano-composites of PEDOT-PSS and MWCNTs, which leads to the improvement of the OLEDs. The nanoparticle $\mathrm{TiO}_{2}$ increased the electron injection at the $\mathrm{TiO}_{2}$-nc film - cathode interface therefore enhancing the internal quantum efficiency. This effect is particularly beneficial for solution processed devices, since these nanoparticles are low cost and easy to handle and might be an alternative to additional polymer layers for controlling charge injection and balance.

\section{REFERENCES}

[1] C. W. Tang and S. A. Van Slyke, Appl. Phys. Lett. 51 (1987) 913.

[2] J. H. Burroughes, D. D. C Bradley, A. R. Brown, R. N. Marks, K. Mackay, R. H. Friend, P. L. Burns and A. B. Holmes, Nature 347 (1990) 608.

[3] J. Kido, K. Hongawa, K. Okuyama and K. Nagai, Appl. Phys. Lett. 64 (1994) 815.

[4] A. R. Brown, N. C. Greenham, J. H. Burroughes, D. D. C. Bradley, R. H. Friend, P. L. Burn, A. Kraft and Holmes A. B. Holmes, Chem. Phys. Lett. 200 (1992) 46.

[5] L. Smilowitz, A. Hays, A. J. Heeger, G. Wang and J. Bowers, E. J. Chem. Phys. 98 (1993) 6504.

[6] Y. Yang and A. Heeger, J. Appl. Phys. Lett. 64 (1994) 1245.

[7] I. D. Parker, J. Appl. Phys. 75 (1994) 1656.

[8] N. P. H. Nam, S. W. Cha, B. S. Kim, S -H. Choi, D. S. Choi and J -I. Jin, Synthetic Metals 130 (2002) 271.

[9] S. J. Chung, J -I. Jin and K. K. Kim, Adv. Mater. 9 (1997) 551.

[10] N. N. Dinh, L. H. Chi, T. T. C. Thuy, T. Q. Trung and V. V. Truong, J. App. Phy. 105 (2009) 1255.

[11] N. N. Dinh, L. H. Chi, T. T. C. Thuy, D. V. Thanh and T. P. Nguyen, J. Korean Phys. Soc. 53 (2008) 802.

[12] T. T. Tung, T. Y. Kim and K. Suh, Organic Electronics 12 (2011) 22-28.

[13] P. H. N. Nguyen and N. D. Nguyen, Adv. Nat. Sci.: Nanosci. Nanotechnol. 2 (2011) 035012.

[14] C. C. Oey, A. B. Djurisic, C. Y. Kwong, C. H. Cheung, W. K. Chan, J. M. Nunzi and P. C. Chui, Thin Solid Films 492 (2005) 253-258.

[15] G. F. Wang, X. M. Tao and R. X. Wang, Composites Science and Technology 68 (2008) 2837-2841.

[16] I. Hulea, R. Van Der Scheer, H. Brom, B. Langeveld-Voss, A. Van Dijken and K. Brunner, Appl. Phys Lett. 83 (2003) 1246.

[17] G. Malliaras, J. Salem, P. Brock and C. Scott, Phys. Rev. B: Condens. Matter 58 (1998) 13411. 